\title{
Correlation between multiple modulation instability side lobes in dispersion oscillating fiber
}

\author{
Xie Wang, ${ }^{1,2}$ Damien Bigourd, ${ }^{1}$ Alexandre Kudlinski, ${ }^{1}$ Kenneth K. Y. Wong, ${ }^{2}$ Marc Douay, ${ }^{1}$ Laurent Bigot, ${ }^{1}$ \\ Antoine Lerouge, ${ }^{1}$ Yves Quiquempois, ${ }^{1}$ and Arnaud Mussot $^{1, *}$ \\ ${ }^{1}$ CNRS-Université Lille 1, PhLAM/IRCICA USR 3380/UMR 8523, F-59655 Villeneuve d'Ascq Cedex, France \\ ${ }^{2}$ Department of Electrical and Electronic Engineering, The University of Hong Kong, Pokfulam Road, Hong Kong \\ ${ }^{*}$ Corresponding author: arnaud.mussot@univ-lille1.fr
}

Received December 19, 2013; revised February 19, 2014; accepted February 23, 2014; posted February 24, 2014 (Doc. ID 203385); published March 21, 2014

\begin{abstract}
We investigate numerically and experimentally the spectral correlation between multiple modulation instability (MI) side lobes in a dispersion oscillating fiber. By leveraging the dispersive Fourier transformation, we acquire instantaneous spectra and investigate the energy correlation between individual MI sidebands through scattergrams. We found that conjugate MI side lobes are strongly correlated while other combinations experience a very low degree of correlation, revealing that parametric processes related to each side lobe pair act quasi-independently. (C) 2014 Optical Society of America

OCIS codes: (190.4410) Nonlinear optics, parametric processes; (190.4380) Nonlinear optics, four-wave mixing; (060.4370) Nonlinear optics, fibers.

http://dx.doi.org/10.1364/OL.39.001881
\end{abstract}

Modulation instability (MI) is one of the most ubiquitous phenomena in nonlinear dynamics, as it appears in many fields of physics [1]. In nonlinear fiber optics, it was discovered more than 30 years ago by Tail et al. [2], and it gave birth to a huge number of investigations, either for fundamental interests or for applications purposes. It is indeed recognized to be at the origin of supercontinuum generation $[\underline{3}, \underline{4}]$, rogue wave formation [5], or wide band optical amplifiers for telecommunication applications, for instance [6]. Basically, MI leads to the exponential amplification of a small perturbation by a strong optical field provided that the nonlinear phase mismatch experienced by the waves is compensated by a linear one [7]. Spectrally, this leads to the formation of two symmetric side lobes around the pump wave. In uniform fibers, this phase-matching relation can be achieved either by pumping in the anomalous dispersion region [2] or in the normal one if the curvature of the group velocity dispersion is negative []. It has also been shown theoretically [9] and experimentally [10] that by periodically modulating the guidance properties of the fiber along the propagation direction, MI is expected to occur whatever the sign of the group velocity dispersion. In that case, one usually refers to a quasiphase matching process that allows the destabilization of many side lobes, more than 10 in a recent experiment [10]. Practically, MI characteristics in the time domain or in the spectral one are recorded with apparatus that require multiple shots to get either autocorrelation trace or average spectrum. While being a noise-driven process, it was then impossible to experimentally investigate its single shot properties experimentally until recently, when dispersive Fourier transformation techniques [11] have been employed to investigate noise-driven nonlinear processes such as rogue wave generation [5], supercontinuum generation [12,13], or MI processes $[14,15]$. In the context of MI, contrary to common belief based on energy conservation laws [7] and averaged spectrum, this new way of investigating MI processes reveals that instantaneous MI spectra can be asymmet- ric [14]. This completely unexpected behavior has been explained through mode competition [14], which of course does not violate the energy conservation relation [7]. All these investigations have been performed in uniform fibers where MI leads to the generation of only two side lobes.

In this Letter, we present a real-time analysis of MI in dispersion oscillating fiber (DOF), where many side lobes can be obtained. Our experimental investigations are based on dispersive Fourier transformation and validated with numerical simulations of stochastic Schrödinger nonlinear equation. The total energy for each MI side lobe is calculated, and their correlation is exhibited by the scattergram. Our results confirm the fundamental property of the MI sidebands in DOF, which is that there is strong positive correlation between conjugate MI sidebands (i.e., the first/second order MI Stokes sideband and the first/second order MI antiStokes sideband) while it reveals that the correlation between distinct MI sidebands (i.e., the first order MI and the second order MI sideband) is much weaker.

The experimental setup is shown in Fig. 1(a). The pump was obtained from a $\mathrm{CW}$ tunable laser source (TLS) at $1554 \mathrm{~nm}$. It was then intensity modulated by a Mach-Zehnder modulator (MZM) to generate 300 ps square-shaped pulses at $4.3 \mathrm{MHz}$ repetition rate. The polarization controllers (PC1) were used to align the state-of-polarization (SOP) of the pump with the transmission axis of the MZM. Afterward, the pump was amplified by two stage erbium-doped fiber amplifiers (EDFA1 and EDFA 2), and two tunable bandpass filters (TBPFs) were inserted to reduce the amplified spontaneous emission (ASE) noise. The PC2 was used to align the SOP of the pump along one of the neutral axis of the fiber. It was finally launched into a $100 \mathrm{~m}$ long DOF. The DOF is a step-index fiber with an average core

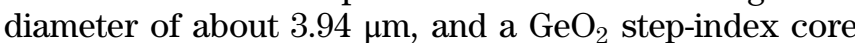
of $23 \times 10^{-3}$. The evolution of the outer diameter of this DOF is represented in Fig. 1(b). It has a longitudinal period of $10 \mathrm{~m}$. By using finite element method 


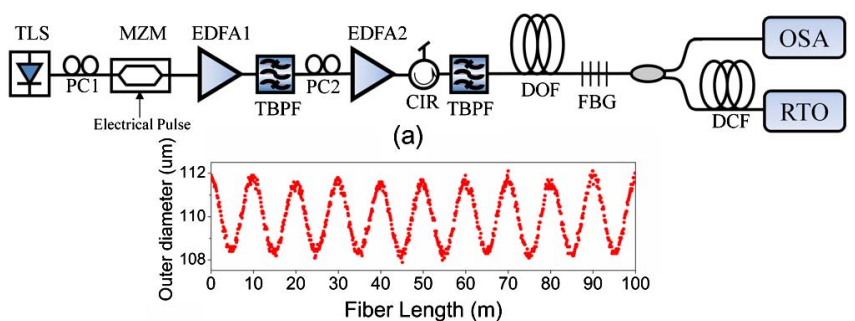

(b)

Fig. 1. (a) Scheme of the experimental setup. TLS, tunable laser source; MZM, Mach-Zehnder modulator; EDFA, erbiumdoped fiber amplifier; TBPF, tunable bandpass filter, PC, polarization controller; CIR, circulator; DOF, dispersion oscillating fiber; DCF, dispersion-compensating fiber; FBG, fiber Bragg grating; OSA, optical spectrum analyzer; RTO, real-time oscilloscope. (b) Longitudinal evolution of the outer diameter of DOF. DOF parameters: average zero dispersion wavelength of $1577 \mathrm{~nm}$, average second-order dispersion $\bar{\beta}_{2}$ of $8.5 \times$ $10^{-4} \mathrm{ps}^{2} / \mathrm{m}$, second-order dispersion amplitude modulation $\beta_{2}^{A}$ of $1.8 \times 10^{-3} \mathrm{ps}^{2} / \mathrm{m}$, average third-order dispersion $\bar{\beta}_{3}$ of $5.2 \times 10^{-5} \mathrm{ps}^{3} / \mathrm{m}$, average fourth-order dispersion $\bar{\beta}_{4}$ of $-1.1 \times 10^{-7} \mathrm{ps}^{4} / \mathrm{m}$, modulation period $Z$ of $10 \mathrm{~m}$, fiber length $L$ of $100 \mathrm{~m}$, and nonlinear coefficient $\gamma$ of $5.5 \mathrm{~W}^{-1} \cdot \mathrm{km}^{-1}$.

simulations, we calculated the DOF parameters that are listed in Fig. 1's caption. A fiber Bragg grating (FBG) was inserted at the output to remove the pump in order to record other much weaker spectral components without saturating the detection setup that was composed either by a conventional optical spectrum analyzer (OSA) or a real-time spectrum analyzer (detailed below). Note that a circulator (CIR) was inserted at the output of EDFA2 to prevent the reflected pump from damaging the equipment.

MI in DOF has been recently investigated experimentally $[10]$ and theoretically $[16,17]$. It has been shown that due to the contribution of the dispersion grating induced by the longitudinal modulation of the group-velocity dispersion (GVD), the following quasi-phase matching relation can be considered to predict the spectral location of MI side lobes:

$$
\bar{\beta}_{2} \Delta \omega^{2}+2 \gamma P_{p}=2 k \pi / Z,
$$

where $\bar{\beta}_{2}$ is the average group velocity dispersion, $\Delta \omega$ is the frequency shift from the pump, $\gamma$ is the nonlinear coefficient, $P_{p}$ is the pump power, $k$ an integer, and $Z$ is the period of modulation. In previous works, we managed to generate more than 10 pairs of MI side lobes [10], but here to focus on investigating the correlation, we adjusted the pump parameters in order to limit the spectrum up to two main MI side lobes with similar gains (corresponding to $k=1$ and $k=2$ respectively). Hence, by launching a pump of $25 \mathrm{~W}$ peak power at $1554 \mathrm{~nm}$, i.e., in slightly normal average dispersion region of the DOF, we obtained the output spectra from conventional OSA represented as dashed-dotted blue line in Fig. 2(a). A set of two symmetric side lobes from the pump has been obtained with a maximum gain difference of less than $6 \mathrm{~dB}$. Note that the noisy spectrum shape of the residual pump is due to the FBG reflection curve, which is not perfectly flat. This spectrum recorded with the OSA remained unchanged from sweep to sweep. However, it does not
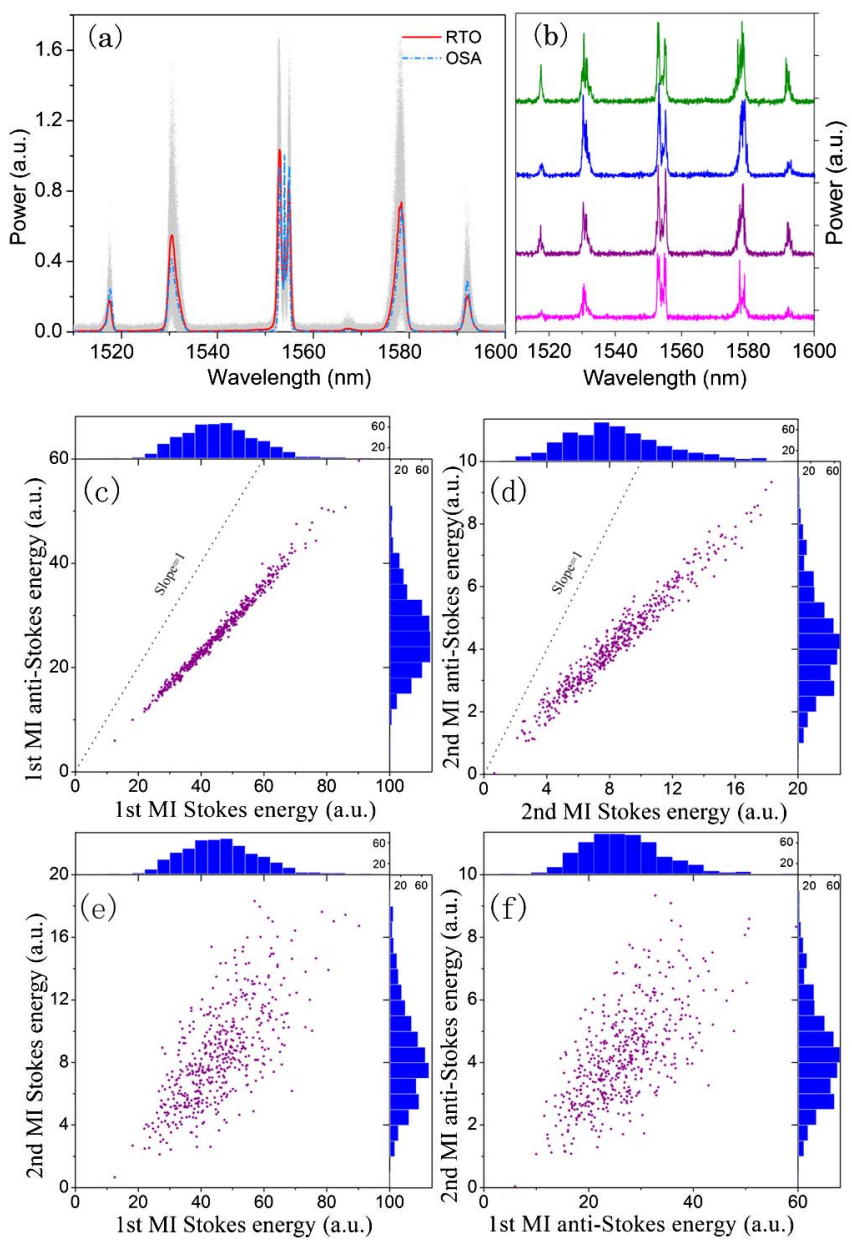

Fig. 2. Experimental results. (a) Spectral measurement from OSA (blue dashed-dotted line), 500 shot-to-shot realizations from RTO measurement (gray dots) and average of 500 realizations from RTO measurement (red solid line). (b) Selected single-shot spectra from RTO measurement illustrating spectral fluctuation. (c) and (d) Scattergrams between conjugate MI sideband, dotted lines with slope of 1 are shown for reference. (e) and (f) Scattergrams between distinct MI sideband.

reveal the real dynamics of the process since shot to shot instabilities are expected to occur because of its noise-driven origin $[\underline{12}, \underline{14}, \underline{18}, \underline{15}]$. In order to investigate the single shot dynamics, a dispersive Fourier transformer has been implemented at the output of the DOF. It consists of a large GVD element (e.g., a DCF) and a real-time oscilloscope (RTO) of $6 \mathrm{GHz}$ bandwidth (photodetector + oscilloscope). After the input optical pulse passing through the dispersive element, the spectrum of the input pulse is mapped to the output temporal waveform. Specifically, the intensity profile of the output pulse is

$$
|U(L, T)|^{2} \sim\left|\tilde{U}\left(0, \frac{-\beta_{2}^{\mathrm{DCF}} L+\sqrt{\left(\beta_{2}^{\mathrm{DCF}} L\right)^{2}+2 \beta_{3}^{\mathrm{DCF}} L T}}{\beta_{3}^{\mathrm{DCF}} L}\right)\right|^{2}
$$

which indicates the following frequency-to-time mapping [11]: 


$$
T(\omega)=\frac{\beta_{3}^{\mathrm{DCF}} L}{2}\left(\omega-\omega_{0}\right)^{2}+\beta_{2}^{\mathrm{DCF}} L\left(\omega-\omega_{0}\right)
$$

where $U$ is the field amplitude, $\widetilde{U}$ is its Fourier transform, $T$ is the time in reference frame of the pulse, $\beta_{2}^{\mathrm{DCF}}$ is the second-order dispersion coefficient in DCF, $\beta_{3}^{\text {DCF }}$ is the third-order dispersion coefficient in DCF, $L$ is the length of the DCF, and $\omega_{0}$ is the center angular frequency of the pulse. Note that in our experiment, due to the relatively large bandwidth, it was necessary to include up to the third-order dispersion term in order to achieve the accurate frequency-to-time conversion. By setting $\beta_{3}^{\mathrm{DCF}}$ to 0 in Eq. (3), we obtain the usual linear mapping expression reported in [12]. With $\beta_{2}^{\mathrm{DCF}} L$ and $\beta_{3}^{\mathrm{DCF}} L$ estimated to be $888 \mathrm{ps}^{2}$ and $-5.4 \mathrm{ps}^{3}$, respectively, we obtain an excellent agreement between the OSA recorded spectrum [dashed-dotted blue trace in Fig. 2(a)] and the average trace recorded with the RTO [red trace in Fig. 2(a)]. Note that the spectrum recorded with the OSA has been vertically scaled owing to the relatively small dynamic range of the real-time spectrum measurement. An ensemble of 500 single-shot time-stretched spectra is superposed as grey dots in Fig. 2(a). We can directly observe the large shot-to-shot fluctuations inside each MI sideband with maximum fluctuation that is larger than twice the average value. In order to get a better insight of these fluctuations, a selection of four single shot spectra is shown in Fig. 2(b). The shape of each MI side lobe is strongly modified compared to the averaged trace, and conjugate MI side lobes are not symmetric from the pump that can be attributed to mode competitions as in [14].

In order to analyze these fluctuations, we numerically calculated the total energy over each experimental MI sidebands by using a numerical square shape filter. We do not try to investigate the peak amplitude variation inside each MI side lobes as it was done in [14] because it was impossible to spectrally resolve each spectral mode in our setup due to the relatively long pump duration used in experiment. This long pulse duration compared to previous studies was imposed by the relatively large frequency shifts of MI side lobes combined to a longer fiber, which requires a minimum pump duration to avoid a walk-off effect between the pump and the side lobes. We defined square shaped filters with a width slightly larger than the MI side lobe under investigation and then obtained one energy value per side lobe per shot. Then we plotted the scattergrams as well as histograms as shown in Fig. 2.

From Figs. $2(\mathrm{c})$ and 2(d), we can observe that data points of energy between conjugate MI sidebands concentrate near a straight line that indicates highly positive correlation between each other [degree of correlation 0.99 and 0.98 for Figs. 2(c) and 2(d), respectively]. Thus we reveal the fact that though there may be a large difference in mode number, mode amplitude, and mode location between conjugate MI sideband as shown in [14], the energy inside each conjugate MI sideband is always highly correlated. We also note the shot-to-shot energy asymmetry between conjugate MI sideband through the scattergram (energy in Stokes sideband is higher than its corresponding anti-Stokes sideband), which is attributed to Raman scattering. The relation between the first-order $\mathrm{MI}$ and second-order $\mathrm{MI}$ is provided in Figs. 2(e) and 2(f); the much weaker correlation is revealed between first-order MI Stokes (anti-Stokes) and second-order MI Stokes (anti-Stokes) (degree of correlation 0.70 and 0.66 , respectively) compared to between conjugate MI sidebands [19]. Through all the experimental results, we may regard the multiple MI processes in DOF as quasi-independent processes.

In order to validate these experimental investigations, we carried out the numerical simulation (400 shot-to-shot realizations) by integrating the stochastic generalized nonlinear Schrödinger equation, taking into account the longitudinal oscillation of the second-order dispersion term only. Indeed, we checked that accounting for the longitudinal evolution of other parameters is negligible. The noisy initial conditions have been simulated by adding half photon per temporal mode [4]. We used the same pulsed pump as in experiments (300 ps duration) except that we slightly adjust its peak power to $23 \mathrm{~W}$ in order to match the experimental MI shape and slightly reduce the fiber length to $80 \mathrm{~m}$ in order to prevent the generation of additional side lobes. As shown in Figs. $\underline{2}$ and $\underline{3}$, the scattergrams and histograms show good
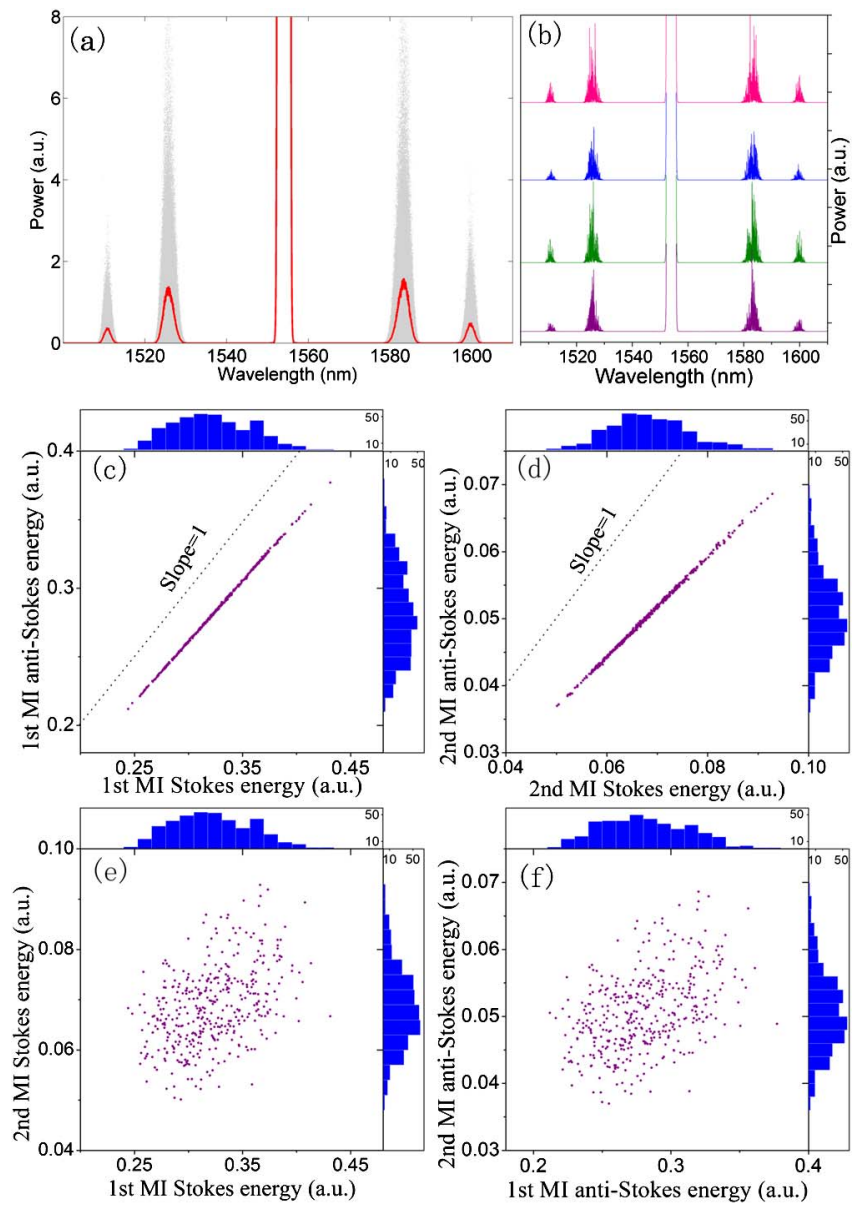

Fig. 3. Numerical simulations. (a) Simulated spectra of the multiple MI sidebands. (b) Selected single-shot spectra. (c) and (d) Scattergrams between conjugate MI sideband, dotted lines with slope of 1 are shown for reference. (e) and (f) Scattergrams between distinct MI sideband. A movie (Media 1) showing the scattergrams evolution along the fiber length can be seen online. 
qualitative agreement between experiment and simulation. The degree of correlation is almost equal to one when considering conjugate side lobes (i.e., 0.99) while they are 0.43 for Figs. 3(e) and 3(f). It also demonstrates that cross combination of MI leads to weak correlation. Moreover, through numerical simulation (see Media 1), we can observe that conjugate MI sidebands become perfectly correlated after propagating $\sim 20 \mathrm{~m}$, while distinct MI sidebands remain quasi-uncorrelated all along the fiber length, though it shows a slight increase of correlation during the propagation. The origin of this low correlation is not fully understood at the present time and it will be investigated elsewhere.

In summary, we have experimentally and numerically investigated the correlation between multiple MI sidebands generated in a DOF. We have verified that conjugate side lobes are strongly correlated, and we have shown that other combination lead to a poor degree of correlation. This reveals that in such kinds of waveguides where multi-parametric processes take place simultaneously, one can consider that they operate quasiindependently. We numerically checked that, first, this could be extended to much more than two QPM side lobes with the same conclusions. Second, as it can be expected, if cascaded FWM side lobes are generated, they are strongly correlated with each other. The following step will consist in performing such kinds of investigations in a nonclassical regime to study the photons-photons correlations properties.

We acknowledge support from the French Agence Nationale de la Recherche (ANR JCJC FOPAFE and TOPWAVE), the Ministry of Higher Education and Research, Nord-Pas de Calais Regional Council and FEDER through the "Contrat de Projets Etat Région (CPER) 2007-2013. The work described in this paper was partially supported by grants from the Research Grants Council of the Hong Kong Special Administrative Region, China (project HKU 717212E).

\section{References and Notes}

1. V. E. Zakharov and L. A. Ostrovsky, Phys. D. 238, 540 (2009).

2. K. Tai, A. Hasegawa, and A. Tomita, Phys. Rev. Lett. 56, 135 (1986).

3. J. M. Dudley, G. Genty, F. Dias, B. Kibler, and N. Akhmediev, Opt. Express 17, 21497 (2009).

4. J. M. Dudley and J. R. Taylor, Supercontinuum Generation in Optical Fibers, 1st ed. (Cambridge University, 2010).

5. D. R. Solli, C. Ropers, P. Koonath, and B. Jalali, Nature 450, 1054 (2007).

6. M. E. Marhic, Fiber Optical Parametric Amplifiers, Oscillators and Related Devices, 1st ed. (Cambridge University, 2007).

7. G. Agrawal, Nonlinear Fiber Optics, 3rd ed. (Academic, 2001).

8. S. B. Cavalcanti, J. C. Cressoni, H. R. da Cruz, and A. S. Gouveia-Neto, Phys. Rev. A 43, 6162 (1991).

9. F. Matera, A. Mecozzi, M. Romagnoli, and M. Settembre, Opt. Lett. 18, 1499 (1993).

10. M. Droques, A. Kudlinski, G. Bouwmans, G. Martinelli, and A. Mussot, Opt. Lett. 37, 4832 (2012).

11. K. Goda, D. R. Solli, K. K. Tsia, and B. Jalali, Phys. Rev. A 80, 043821 (2009).

12. B. Wetzel, A. Stefani, L. Larger, P. A. Lacourt, J. M. Merolla, T. Sylvestre, A. Kudlinski, A. Mussot, G. Genty, F. Dias, and J. M. Dudley, Sci. Rep. 2, 882 (2012).

13. T. Godin, B. Wetzel, T. Sylvestre, L. Larger, A. Kudlinski, A. Mussot, A. Ben Salem, M. Zghal, G. Genty, F. Dias, and J. M. Dudley, Opt. Express 21, 18452 (2013).

14. D. R. Solli, G. Herink, B. Jalali, and C. Ropers, Nat. Photonics 6, 463 (2012).

15. A. Mussot and A. Kudlinski, Nat. Photonics 6, 415 (2012).

16. M. Droques, A. Kudlinski, G. Bouwmans, G. Martinelli, and A. Mussot, Phys. Rev. A 87, 013813 (2013).

17. A. Armaroli and F. Biancalana, Opt. Express 20, 25096 (2012).

18. D. R. Solli, C. Ropers, and B. Jalali, Nonlinearity 26, R85 (2013).

19. Note that here we do not plot the scattergram between first-order MI Stokes (anti-Stokes) and second-order MI anti-Stokes (Stokes) as it would be similar to the ones depicted in Figs. 2(e) and 2(f) due to the high degree of correlation between conjugate MI sidebands. 